\title{
Unfocused Extracorporeal Shock Waves Induce Anabolic Effects in Rat Bone
}

By O.P. van der Jagt, MD, T.M. Piscaer, MD, W. Schaden, J. Li, PhD, N. Kops, H. Jahr, PhD, J.C. van der Linden, PhD,

J.H. Waarsing, PhD, J.A.N. Verhaar, MD, PhD, M. de Jong, PhD, and H. Weinans, PhD

Investigation performed at the Department of Orthopaedics, Erasmus MC, University Medical Center Rotterdam, The Netherlands

Background: Extracorporeal shock waves are known to stimulate the differentiation of mesenchymal stem cells toward osteoprogenitors and induce the expression of osteogenic-related growth hormones. The aim of this study was to investigate if and how extracorporeal shock waves affected new bone formation, bone microarchitecture, and the mechanical properties of bone in a healthy rat model, in order to evaluate whether extracorporeal shock wave therapy might be a potential treatment for osteoporosis.

Methods: Thirteen rats received 1000 electrohydraulically generated unfocused extracorporeal shock waves to the right tibia. The contralateral, left tibia was not treated and served as a control. At two, seven, twenty-one, and forty-nine days after administration of the shock waves, in vivo single-photon-emission computed tomography (SPECT) scanning was performed to measure new bone formation on the basis of uptake of technetium-labeled methylene diphosphonate (99mTc-MDP) $(n=6)$. Prior to and forty-nine days after the extracorporeal shock wave therapy, micro-computed tomography (micro-CT) scans were made to examine the architectural bone changes. In addition, mechanical testing, microcrack, and histological analyses were performed.

Results: Extracorporeal shock waves induced a strong increase in 99mTc-MDP uptake in the treated tibia compared with the uptake in the untreated, control tibia. Micro-CT analysis showed that extracorporeal shock waves stimulated increases in both trabecular and cortical volume, which resulted in higher bone stiffness compared with that of the control tibiae. Histological analysis showed intramedullary soft-tissue damage and de novo bone with active osteoblasts and osteoid in the bone marrow of the legs treated with extracorporeal shock waves. Microcrack analysis showed no differences between the treated and control legs.

Conclusions: This study shows that a single treatment with extracorporeal shock waves induces anabolic effects in both cancellous and cortical bone, leading to improved biomechanical properties. Furthermore, treatment with extracorporeal shock waves results in transient damage to the bone marrow, which might be related to the anabolic effects. After further examination and optimization, unfocused extracorporeal shock waves might enable local treatment of skeletal sites susceptible to fracture.

Clinical Relevance: Unfocused extracorporeal shock waves might in the future be used to increase bone mass and subsequently reduce the fracture risk.

C urrent pharmaceutical treatments for osteoporosis interfere in the remodeling cycle by blocking osteoclastic resorption or changing the balance between bone resorption and formation. These therapies require regular intake of medication and are accompanied by potential adverse side effects. Biophysical stimuli such as mechanical vibration, ultrasound, and pulsed electromagnetic fields have been sug- gested as an alternative treatment, but these stimuli seem to have too little effect on bone remodeling and bone architecture ${ }^{1-4}$. Another potentially useful biophysical intervention that has proven effective for acute fractures ${ }^{5}$ and nonunions ${ }^{6-8}$ is treatment with extracorporeal shock waves.

Shock waves are acoustical pulses that are characterized by a high amplitude ( $\leq 120 \mathrm{MPa})$ and a short rise time ( $\leq 10 \mathrm{~ns})$

Disclosure: In support of their research for or preparation of this work, one or more of the authors received, in any one year, a research grant in excess of $\$ 10,000$ from the Dutch Technology Foundation STW. In addition, one or more of the authors or a member of his or her immediate family received, in any one year, payments or other benefits in excess of $\$ 10,000$ or a commitment or agreement to provide such benefits from a commercial entity (Tissue Regeneration Technologies). 
The Journal of Bone \& Joint Surgery - Jbjs.org Volume 93-A • Number 1 · January 5, 2011
Unfocused Extracorporeal Shock WaVes

Induce ANabolic EFfects in Rat Bone and are followed by a longer low-magnitude negative wave $(\leq 10 \mathrm{MPa})^{9}$.

Focused shock waves have been reported to alter bone turnover or induce trabecular or cortical bone formation in animal models ${ }^{10-12}$. Several mechanisms for triggering these anabolic effects have been suggested. First, the increased bone formation is associated with an increased expression of several growth factors (including bone morphogenetic proteins [BMPs], transforming growth factor-beta [TGF- $\beta$ ], and vascular endothelial growth factors [VEGFs]) and the activation of cell signaling pathways like ERK and $\mathrm{Wnt}^{13-17}$. Second, shock waves stimulate both the recruitment and the differentiation of mesenchymal stem cells $s^{13,14,18}$. Finally, it is suggested that the effects are triggered by damage, since periosteal detachment, cortical fractures, and disruptions of trabeculae are observed after application of extracorporeal shock wave treatment ${ }^{10,19,20}$.

In all of the experimental studies cited above, the investigators applied focused shock waves to a small area. However, since the skeletal sites that need to be treated in osteoporosis are much larger than the area encompassed by focused shock wave therapy, it is difficult to apply focused shock wave therapy for the treatment of osteoporosis. Transducers that apply unfocused shock waves are now available. These transducers have a treatment zone of $3.8 \mathrm{~cm}$ in diameter, allowing treatment of skeletal sites that are prone to fracture in patients with osteopenic conditions. However, it is unknown whether, and for how long, unfocused shock waves can influence bone remodeling, bone microarchitecture, and the mechanical properties of bone.

While the overall research aim is to explore the possibilities of using extracorporeal shock waves for osteoporosis therapy, the purpose of this particular study was to investigate whether, and how, unfocused extracorporeal shock wave therapy affects new bone formation, bone microarchitecture, and the mechanical properties of bone in a healthy rat model.

\section{Materials and Methods}

\section{Animals and Treatment Protocol}

Six male Wistar rats (fifteen weeks of age) obtained from Harlan Laboratories (Horst, The Netherlands) underwent extracorporeal shock wave therapy and were followed for fortynine days with single-photon-emission computed tomography (SPECT) scanning and in vivo micro-computed tomography (micro-CT) scanning. Seven additional rats were used in other experiments to examine osseous and soft-tissue microdamage. Those seven animals were killed twenty-four hours after administration of the extracorporeal shock wave therapy. The six rats used for ongoing study were housed sometimes in pairs and sometimes in threesomes at the animal facility of the Erasmus $\mathrm{MC}$, with a twelve-hour light-dark regimen, at $21^{\circ} \mathrm{C}$. The animals received standard food pellets and water ad libitum. The research protocol (number 116-07-02) was approved by the local committee for animal experiments and is in accordance with Dutch law.

The sample size was chosen for logistical reasons and not on the basis of a priori hypothesized effect-size and variance estimates. Thus, the analyses are exploratory, and effects that were not found to be significant should not be presumed to be clinically unimportant. These data provide effect and variance estimates for future work.

\section{Extracorporeal Shock Wave Therapy}

Both hind legs of the anesthetized rat were shaved, and the rat was placed on its left dorsolateral side. An ultrasonic gel that served as coupling medium was applied to the right hind leg. The lithotripter of the shock wave device (Dermagold; Tissue Regeneration Technologies, Woodstock, Georgia, manufactured by MTS, Konstanz, Germany) was placed at the anterolateral side of the right hind leg and covered the whole tibia. A total of 1000 electrohydraulically generated extracorporeal shock waves were applied at $3 \mathrm{~Hz}$ with an energy flux density of $0.3 \mathrm{~mJ} / \mathrm{mm}^{2}$. The contralateral, left tibia served as a control and was not treated.

\section{Multi-Pinhole SPECT Scanning}

At two, seven, twenty-one, and forty-nine days after the extracorporeal shock waves were applied, the local bone formation was analyzed with use of multi-pinhole (mph) SPECT ${ }^{21}$. At four hours prior to scanning, $185-\mathrm{MBq}(4995-\mu \mathrm{Ci})$ technetiumlabeled methylene diphosphonate ( $\left.{ }^{99 \mathrm{~m}} \mathrm{Tc}-\mathrm{MDP}\right)$ was intravenously injected. ${ }^{99 \mathrm{~m}} \mathrm{Tc}-\mathrm{MDP}$ first binds to the unmineralized extracellular matrix of newly formed bone and is subsequently irreversibly incorporated into the mineralized extracellular matrix. The animals were scanned with use of a dedicated small-animal mph-SPECT scanner (Nanospect; Bioscan, Washington, DC). During scanning, the animals were anesthetized with isoflurane (2\%). To minimize interference with the radioactive signal from the body, the hind legs were stretched and were fixed in a custom-made wooden frame so that both tibiae could be scanned at the same time.

The total scanning time was twenty-six minutes. Four gamma cameras, each containing nine pinholes of $2.5 \mathrm{~mm}$ in diameter, were used. Acquired scan data were reconstructed at a voxel size of $0.33 \mathrm{~mm}$. The images were rotated so that all tibiae appeared with the same orientation. Two regions of interest were determined in the reconstructed image: the first $(5 \mathrm{~mm}$ in width) was positioned $1 \mathrm{~mm}$ distal to the active signal of the growth plate, and the second ( $7 \mathrm{~mm}$ in width) was positioned at the diaphysis $8 \mathrm{~mm}$ distal to the growth plate (Fig. 1). The amount of radioactive tracer uptake was quantified within each region of interest.

\section{In Vivo Micro-CT Scanning}

Prior to and forty-nine days after extracorporeal shock wave therapy, in vivo micro-CT scans were made with the animal under anesthesia. The hind leg of the rat was fixed in a supine position, allowing a $17-\mathrm{mm}$ scan of the proximal part of the tibia. Scanning with a Skyscan 1076 Scanner (Kontich, Belgium) was performed at a voltage of $60 \mathrm{kV}$, at a current of $167 \mu \mathrm{A}$, and with use of a $0.5-\mathrm{mm}$ aluminium filter, over $196^{\circ}$ with a rotation step of $1^{\circ}$, with each scan taking eight minutes. This resulted in data sets with an isotropic voxel size of $18 \mu \mathrm{m}$. 


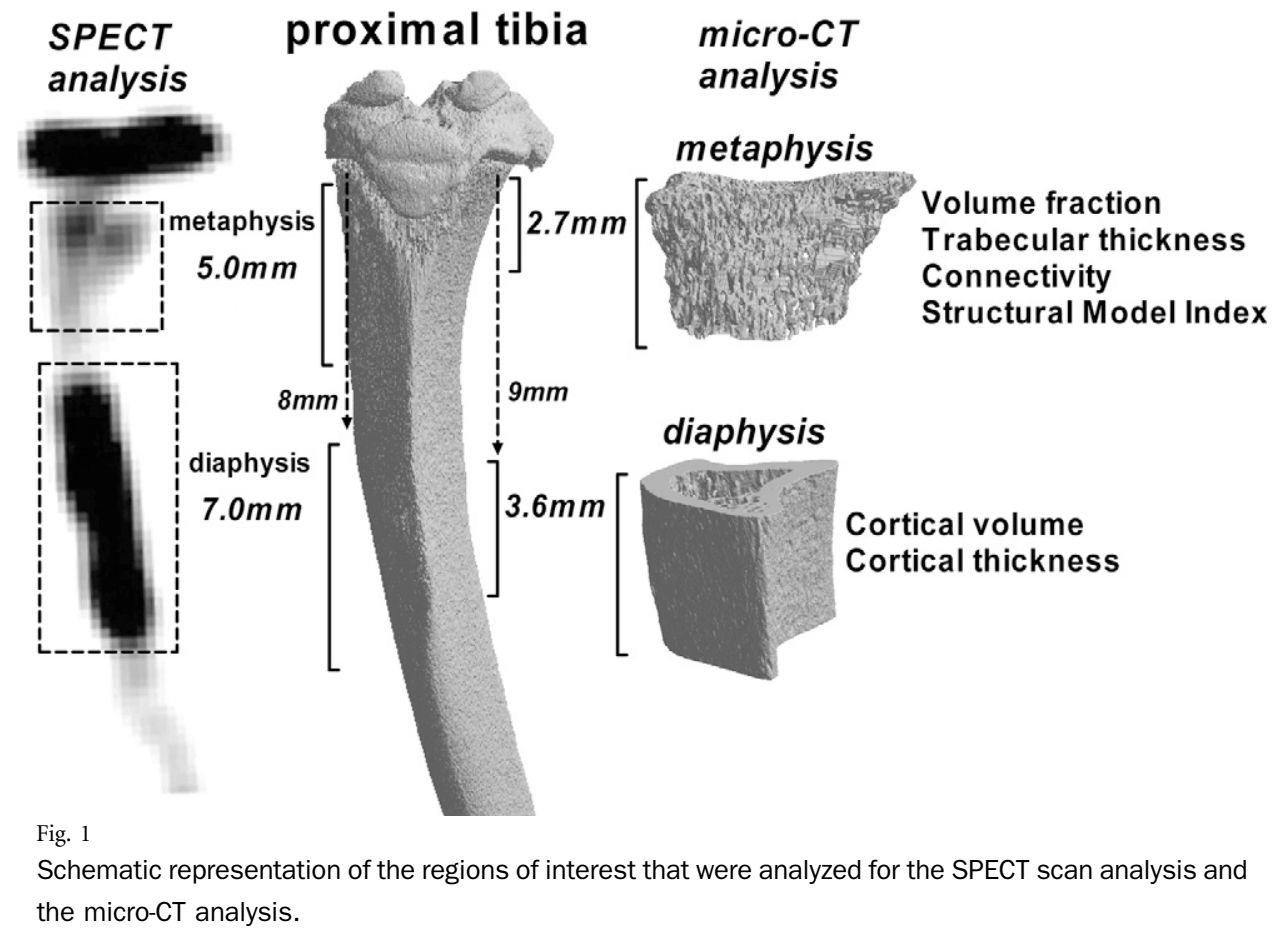

NRecon software (version 1.5, Skyscan) was used to make three-dimensional reconstructions of two regions of interest, similar to the regions of interest for the mph-SPECT scans. The reconstruction of the proximal metaphysis included the region starting just distal to the physis and continued distally for $2.7 \mathrm{~mm}$ (Fig. 1). The reconstruction of the diaphysis was defined by a region of $3.6 \mathrm{~mm}$, starting $9 \mathrm{~mm}$ distal to the physis. Osseous and nonosseous structures were separated with use of a local threshold algorithm (software freely available) ) $^{22}$ resulting in binary data sets ${ }^{23}$. Cortical and trabecular bone were automatically separated with use of in-house software. Trabecular architecture in the proximal metaphysis was characterized by determining the trabecular volume fraction (trabecular bone volume divided by total volume), connectivity density (connectivity divided by total volume), structural model index (in which an index of 3 indicates the presence of rods and an index of 0 indicates the presence of plates), and three-dimensional trabecular thickness. Cortical architecture was assessed in the diaphysis and was characterized by cortical volume, three-dimensional cortical thickness, and the periosteal and endosteal perimeters.

\section{Three-Point Bending Tests}

Mechanical properties were determined with use of three-point bending. At day 49, the animals were killed with use of an overdose of pentobarbital. The tibiae were dissected, the soft tissue was removed, and the specimens were stored in phosphatebuffered saline solution at $4^{\circ} \mathrm{C}$. Testing with a Single Column Lloyd LRX Testing System (Lloyd Instruments, Hampshire, United Kingdom) was performed with the bones equilibrated to room temperature. Each tibia was stably positioned on its medial anterolateral site. The distance between the loading posts was $2.36 \mathrm{~cm}$; in this way, one post was just distal to the condyles and the other was just proximal to the malleoli. To ensure a stable position of the bone, five preconditioning runs with a displacement rate of $0.01 \mathrm{~mm} / \mathrm{s}$ and a maximal force of $4 \mathrm{~N}$ were made, after which the load returned to the position corresponding to $0 \mathrm{~N}^{24}$. Thereafter, the fracture test was carried out with a displacement rate of $0.01 \mathrm{~mm} / \mathrm{s}$ and a maximal displacement of $3.0 \mathrm{~mm}$. Displacement $(\mathrm{mm})$ and force $(\mathrm{N})$ were registered at a sample rate of $20 \mathrm{~Hz}$. Force displacement graphs were made, and load to failure, toughness, stiffness, and the Young modulus were determined. The Young modulus was determined with the equation $\mathrm{E}=\mathrm{F} / \mathrm{D}^{\star} \mathrm{l}^{3} / \mathrm{I}_{\mathrm{x}}$, in which $\mathrm{F} / \mathrm{D}$ is the stiffness, $l$ is the length between the loading posts, and $\mathrm{I}_{\mathrm{x}}$ is the moment of inertia around the bending axis. The moment of inertia was determined from the micro-CT cross section that corresponded with the fracture site. This cross section was rotated so that the moment of inertia around the bending axis $\left(\mathrm{I}_{\mathrm{x}}\right)$ could be calculated with CT analyzer software (Skyscan).

\section{Histological Analysis}

Directly after mechanical testing the hind legs were fixed in $4 \%$ paraformaldehyde. After twenty-one days, the proximal halves of the tibiae were dehydrated and were block-embedded in methylmethacrylate. Sagittal sections ( $6 \mu \mathrm{m}$ thick) were made of the entire proximal part of the tibia. The overall appearance was evaluated with hematoxylin and eosin $(\mathrm{H} \& \mathrm{E})$ staining (Gill hematoxylin for five minutes and eosin for one minute), and new bone formation was evaluated with use of thionine staining $(0.05 \%$ thionine in $0.01 \mathrm{M}$ aqueous sodium phosphate, $\mathrm{pH} 5.8$, for five minutes). To evaluate the bone marrow 
The Journal of Bone \& Joint Surgery · Jbjs.org Volume 93-A • Number 1 · January 5, 2011
Unfocused Extracorporeal Shock Waves

Induce ANabolic EfFects in Rat Bone specifically, May-Grünwald-Giemsa staining was performed (May-Grünwald stain for five minutes and Giemsa stain for twenty minutes). The number of adipocytes was counted in an area of $2.25 \mathrm{~cm}^{2}$ in three different matched sections of treated and control samples. Accordingly, the diameter of fifty adipocytes was measured.

Perls staining to detect the presence of hemosiderin (an indication that bleeding has occurred) was performed by incubating the sections in $2 \%$ hydrochloric acid and $2 \%$ potassium hexacyanoferrate (Klinipath, Duiven, The Netherlands) for twenty minutes. After they were rinsed in distilled water, the samples were counterstained with pararosaniline $(0.02 \%)$.

To analyze the presence of periosteal detachment, subperiosteal hemorrhage, and intramedullary damage, three additional rats were administered extracorporeal shock wave therapy as described above. At twenty-four hours after the extracorporeal shock wave therapy, both the treated and the untreated hind legs were harvested and processed as described above. $\mathrm{H} \& \mathrm{E}$ and Perls staining was then performed.

\section{Microcrack Analysis}

To assess whether extracorporeal shock wave therapy had induced microdamage to the bone, four additional animals were obtained. Again, extracorporeal shock wave therapy was applied to one leg, and the contralateral (untreated) leg served as the control. At twenty-four hours after extracorporeal shock wave therapy, the animals were killed and the tibiae were dissected and fixed in formaldehyde (4\%).

The tibiae were stained with en bloc basic fuchsin staining and embedded in methylmethacrylate ${ }^{25}$. The proximal part of the tibia was cut in $150-\mu$ m-thick frontal sections with use of a diamond wire saw (Histo Saw; Delaware Diamond Knives, Wilmington, Delaware). Bone measurement was performed at $200 \times$ magnification of an 8 to $10-\mathrm{mm}^{2}$ region of secondary spongiosa $1 \mathrm{~mm}$ away from the growth plate with use of the Bioquant digitizing system (R\&M Biometrics, Nashville, Tennessee). Stained microcracks were defined by sharp edges, some depth of field, and permeation of stain into the crack walls. Measurements included crack density (crack number $/ \mathrm{mm}^{2}$ of bone area), mean crack length (in $\mu \mathrm{m}$ ), and crack surface density (crack number $\times$ crack length/bone area, in $\mu \mathrm{m} / \mathrm{mm}^{2}$ ).

\section{Statistical Methods}

Statistical analysis was performed with GraphPad Prism Software (GraphPad Software, San Diego, California), with use of paired testing, in which the treated and untreated (control) legs of each animal served as a pair. The results of the mph-SPECT scans were statistically analyzed with use of the Wilcoxon matched-pairs test, since the uptake of radionuclide was not normally distributed. All other analyzed parameters were
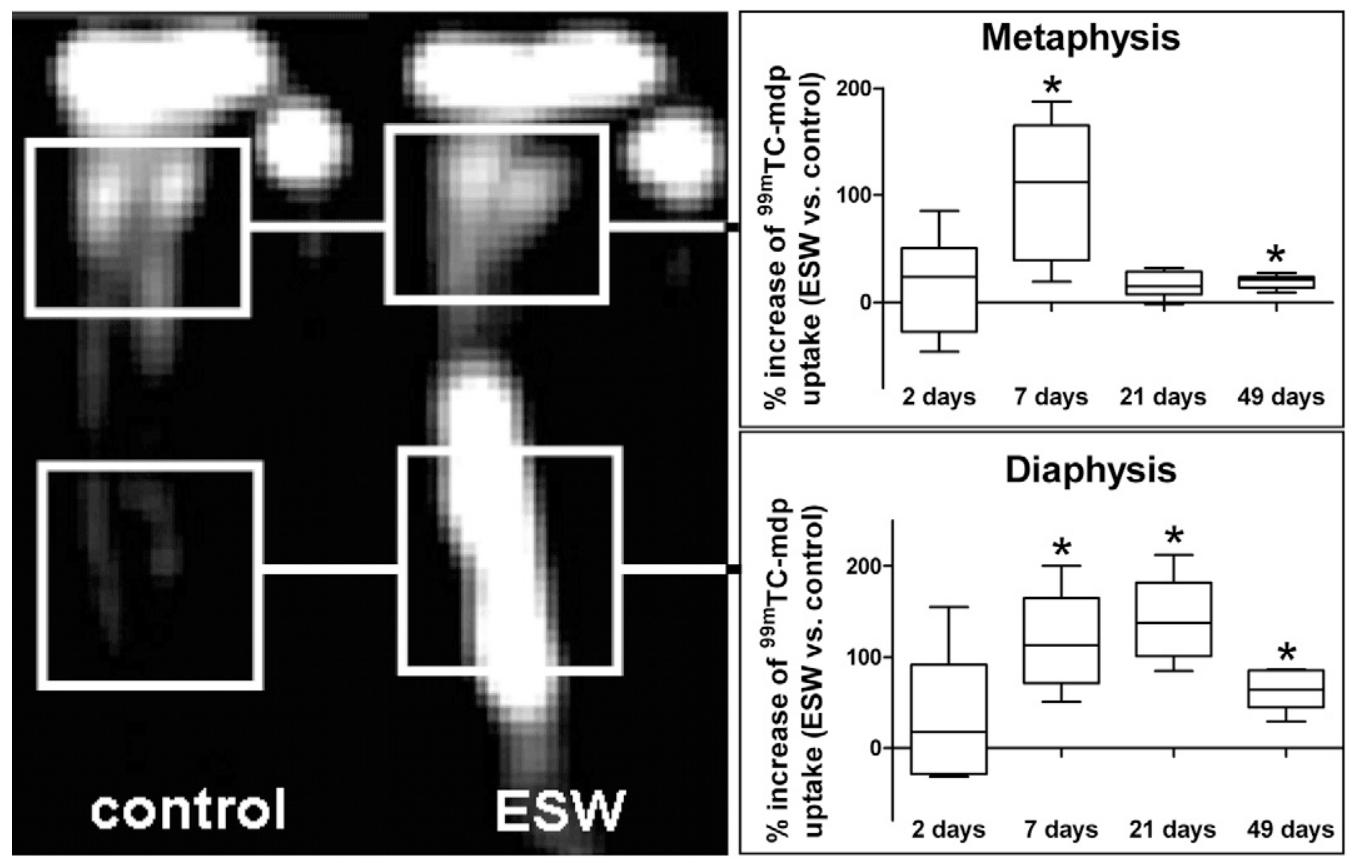

Fig. 2

Representative SPECT scans, made at twenty-one days, of a control tibia and a tibia treated with extracorporeal shock waves (ESW). The regions of interest are indicated and are attached to box-and-whisker plots in which the

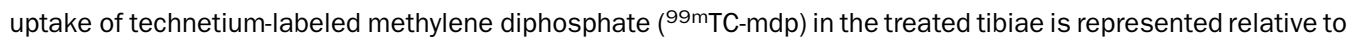
the uptake in the controls at different time points. $* P<0.05$. The boxes represent the $5 \%$ to $95 \%$ confidence intervals, the horizontal lines within the boxes represent the median, and the I bars represent the minimum and maximum values. 


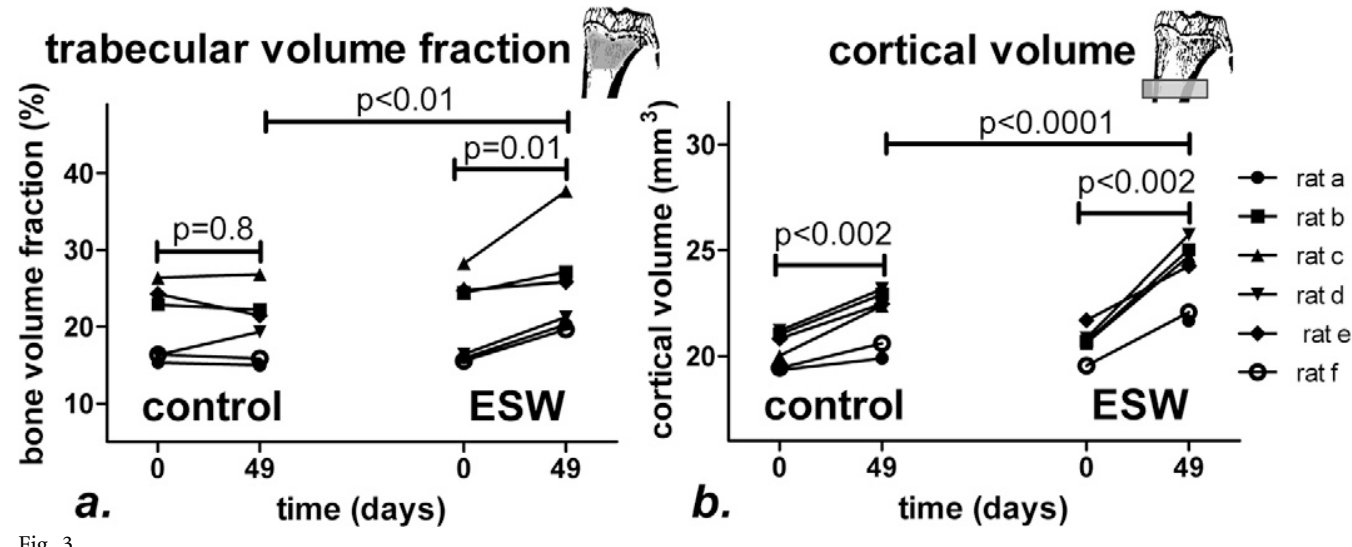

Trabecular volume fraction (a) and cortical volume (b) for the extracorporeal shock wave (ESW)-treated and control tibiae of all rats. The $p$ values for the differences between the treated and untreated tibiae at day 49 and the $p$ values for the differences between day 0 and day 49 are indicated.

normally distributed, and statistical analysis was performed with use of paired $t$ tests. In the analysis of the results of the micro-CT scanning, differences between the findings at the start and the end of the experiment were also assessed with use of paired $t$ tests, in which two time points (day 0 [prior to the extracorporeal shock wave therapy] and day 49) of one leg served as a pair.

\section{Source of Funding}

Funding for this study was received from the Dutch Technology Foundation STW (RPG 6294), which played no role in the study.

\section{Results}

C xtracorporeal shock wave therapy caused redness of the skin -1 and minor superficial bleeding; however, fifteen minutes
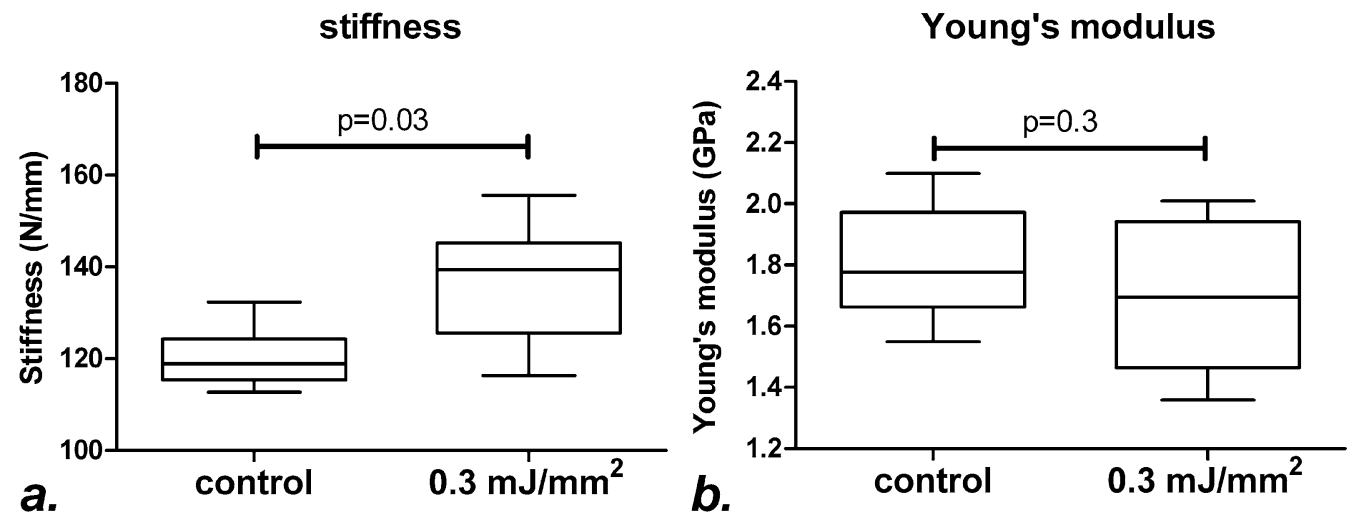

maximal Force

\section{toughness}
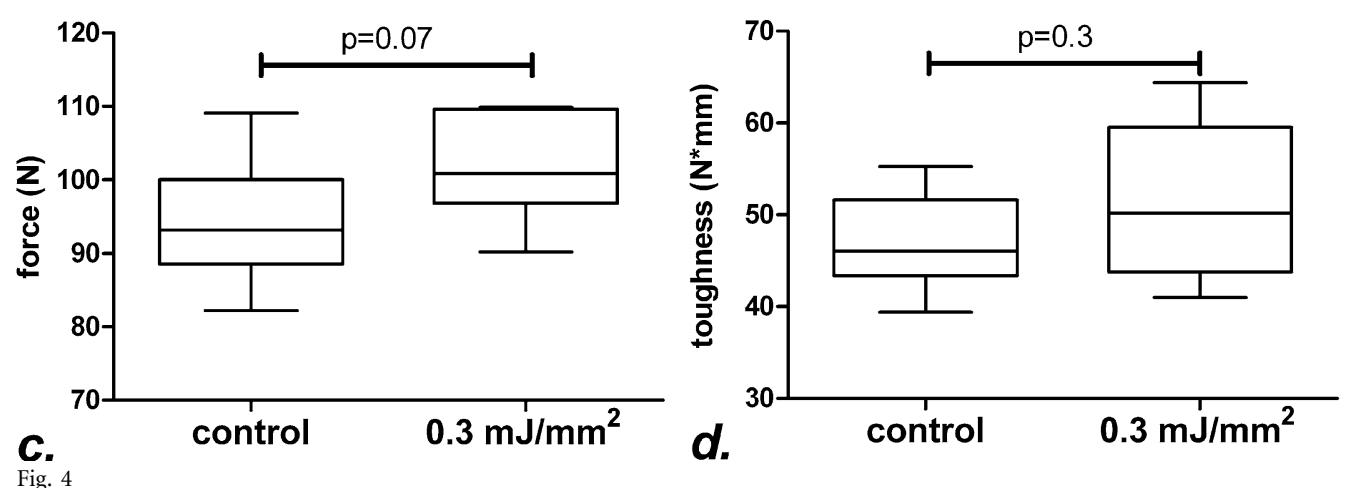

Box-and-whisker plots of the stiffness, Young modulus, maximal force, and toughness in the control and extracorporeal shock wave-treated $\left(0.3 \mathrm{~mJ} / \mathrm{mm}^{2}\right)$ tibiae. The boxes represent the $5 \%$ to $95 \%$ confidence intervals, the horizontal lines within the boxes represent the median, and the I bars represent the minimum and maximum values. 
The Journal of Bone \& Joint Surgery · Jbjs.org Volume 93-A • Number 1 · January 5, 2011
Unfocused Extracorporeal Shock WaVes

Induce ANabolic EFfects in Rat Bone after the therapy, all animals had recovered from the anesthesia, were using the treated leg, and behaved normally. During the experimental period, no animals showed weight loss. The average weight gain during this forty-nine-day study period was $63 \mathrm{~g}$ (range, 54 to $73 \mathrm{~g}$ ).

\section{MPH-SPECT Scanning}

Extracorporeal shock wave therapy induced an increased uptake of ${ }^{99 \mathrm{~m}} \mathrm{Tc}-\mathrm{MDP}$ in the treated tibiae compared with the untreated, control tibiae. At two days after the extracorporeal shock wave therapy, the treated legs showed a nonsignificant increase in uptake of ${ }^{99 \mathrm{~m}} \mathrm{Tc}-\mathrm{MDP}$. At seven days, the increase in the metaphysis was more than twofold compared with the uptake in the untreated legs. At twenty-one and forty-nine days, the difference in uptake between the treated and untreated legs had decreased, although at forty-nine days the treated legs still showed $20 \%$ more uptake compared with the uptake in the untreated legs (Fig. 2).

At two days after extracorporeal shock wave therapy, the uptake of ${ }^{99 \mathrm{~m}} \mathrm{Tc}-\mathrm{MDP}$ in the diaphysis showed a nonsignificant increase in the treated legs compared with that in the untreated legs. At seven days, the uptake in the treated legs was more than twice that in the untreated legs. This highly increased uptake was also observed at twenty-one days. At forty-nine days, the treated legs still showed $63 \%$ more uptake than the untreated legs (Fig. 2).

\section{Microarchitectural Bone Changes}

The trabecular volume fraction of the treated legs increased from $20.5 \% \pm 5.55 \%$ (mean and standard deviation) at day 0 to $25.3 \% \pm 6.77 \%$ at day $49(\mathrm{p}=0.01)$ (Fig. $3, a)$, whereas the trabecular volume fraction of the untreated, control legs $(20.3 \% \pm 4.8 \%$ at day 0 and $20.1 \% \pm 4.37 \%$ at day $49 ; \mathrm{p}=0.8)$ did not change significantly during this period. At day 49 , all treated legs had a significantly higher trabecular bone volume than the untreated legs $(\mathrm{p}<0.01)$.

At day 49, the connectivity density in the treated legs was $41.9 \pm 14.1$ compared with $27.7 \pm 6.4$ in the untreated, control legs $(\mathrm{p}=0.02)$. There was a difference between the structural model index in the treated legs $(1.9 \pm 0.28)$ and that in the untreated legs $(2.1 \pm 0.21)(\mathrm{p}=0.03)$, indicating that the trabeculae of the treated legs were more plate-like than those of the untreated, control legs. No significant difference in trabecular thickness was found between the treated and untreated legs.

The cortical volume of the treated legs increased from $20.7 \pm 0.77 \mathrm{~mm}^{3}$ at day 0 to $23.9 \pm 1.66 \mathrm{~mm}^{3}$ at day 49 (Fig. 3, $b$ ), whereas the cortical volume of the untreated, control legs increased from $20.3 \pm 0.83 \mathrm{~mm}^{3}$ to $21.9 \pm 1.34 \mathrm{~mm}^{3}$ during this period. At day 49, the cortical volume of all treated legs was higher than that of the control legs $(\mathrm{p}<0.0001)$. The mean three-dimensional thickness of the cortex increased from $678 \pm$ $39 \mu \mathrm{m}$ to $767 \pm 78 \mu \mathrm{m}$ in the treated legs $(\mathrm{p}<0.01)$ and increased from $670 \pm 43 \mu \mathrm{m}$ to $714 \pm 52 \mu \mathrm{m}$ in the untreated legs $(\mathrm{p}<0.002)$. At day 49 , the mean cortical thickness of the treated legs was significantly higher than that of the untreated control legs $(\mathrm{p}<0.01)$.
At day 49, measurement of the cortical perimeters showed no significant difference in the mean endosteal perimeter between the treated and untreated legs $(8.6 \pm 0.69 \mathrm{~mm}$ and $8.5 \pm 0.68 \mathrm{~mm}$, respectively; $\mathrm{p}=0.8$ ). The periosteal perimeter in the treated legs was significantly higher than that in the untreated legs $(15.49 \pm 0.54 \mathrm{~mm}$ and $15.06 \pm 0.48 \mathrm{~mm}$, respectively; $\mathrm{p}=0.03$ ), suggesting that new bone formation mainly occurred at the periosteal side of the cortex.

\section{Mechanical Testing}

Three-point bending tests showed that the stiffness of the treated tibiae was significantly higher than that of untreated, control tibiae $(137 \pm 13.2 \mathrm{~N} / \mathrm{mm}$ and $120 \pm 6.7 \mathrm{~N} / \mathrm{mm}$, respectively; $\mathrm{p}=0.03$ ) (Fig. $4, a$ ). The Young modulus did not differ significantly between the treated and untreated legs (1.7 \pm $0.2 \mathrm{GPa}$ and $1.8 \pm 0.2 \mathrm{GPa}$, respectively; $\mathrm{p}=0.3$ ), suggesting that the difference in geometry and increased cortical bone volume

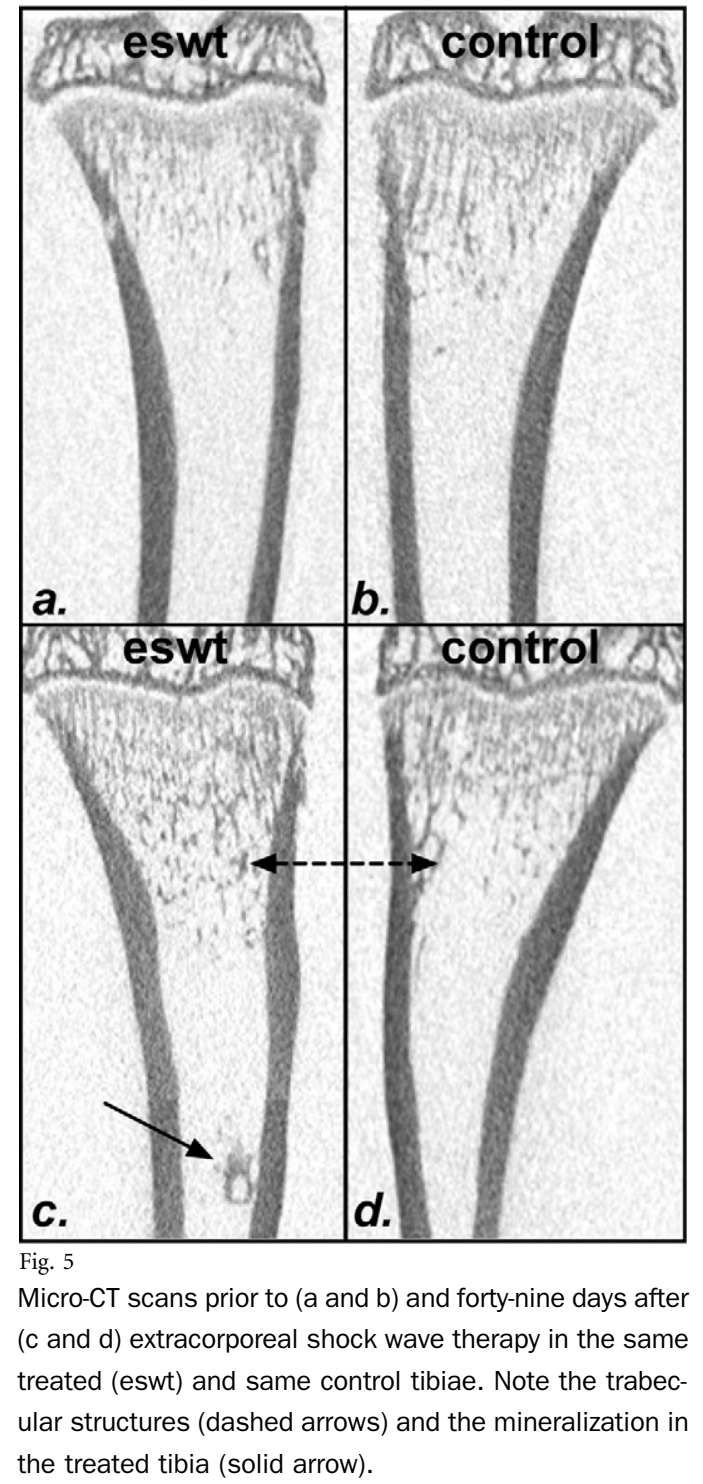


The Journal of Bone \& Joint Surgery - JBjs.org Volume 93-A • Number 1 · January 5, 2011
Unfocused Extracorporeal Shock Waves

Induce Anabolic EfFects in Rat Bone explain the increased stiffness in the treated legs (Fig. 4, $b$ ). The maximal force did not differ significantly between the treated and untreated legs $(101.7 \pm 7.3 \mathrm{~N}$ and $94.2 \pm 7.3 \mathrm{~N}$, respectively; $\mathrm{p}=0.07$ ) (Fig. 4, $c$ ), and no significant difference in toughness was found between the treated and untreated tibiae (51.4 \pm $8.8 \mathrm{~N} \cdot \mathrm{mm}$ and $47.0 \pm 5.4 \mathrm{~N} \cdot \mathrm{mm}$, respectively; $\mathrm{p}=0.3)($ Fig $4, d)$. There was also no difference in the fracture location. All tibiae fractured at the site of the loading application.

\section{Bone Changes on Micro-CT Scans and Histological Analysis} Examination of the micro-CT scans showed that the region of the proximal metaphysis that was filled with trabecular bone was larger in the treated legs (an average of $5.8 \mathrm{~mm}$ from the epiphysis) than in the untreated, control legs $(4.2 \mathrm{~mm}$ ) (Fig. 5). Furthermore, mineralization in the midpart of the diaphysis was observed in the treated legs (Fig. 5, d). Histological examination showed that these mineralizations were present around fibrotic tissue and had an osseous morphology with osteocytes, suggesting de novo bone formation (Fig. 6, $a$ ). Active osteoblasts and osteoid were observed in these ossifications (Fig. 6, $b$ ). In the cortex, cement lines at the periosteal site, indicating periosteal apposition, could be identified (Fig. 6, c). The periosteum had a normal morphology at forty-nine days (Fig. 6, $d$ ).

In the hind legs harvested twenty-four hours after extracorporeal shock wave therapy, necrotic hematopoietic pro- genitors and cell debris were observed throughout the bone marrow (Fig. 7, $a$ and $b$ ). Furthermore, adipocytes were damaged, characterized by the disruption of cell membranes (Fig. 7, $a$ and $b$ ). At forty-nine days, hematopoietic progenitors were reestablished in the bone marrow (Fig. 7, $c$ and $d$ ). The adipocyte number and size were, however, higher in the treated legs than in the untreated, control legs at forty-nine days (Fig. $7, e$ and $f$ ). The mean number of adipocytes in the treated and untreated legs was $120.4 \pm 18.2$ and $69.2 \pm 25.6$, respectively $(\mathrm{p}=0.003)$. The adipocytes in the treated legs had a mean diameter of $0.04 \pm 0.004 \mathrm{~mm}$ compared with $0.02 \pm 0.003 \mathrm{~mm}$ in the untreated legs $(\mathrm{p}=0.0002)$. At forty-nine days, Perls staining showed deposition of hemosiderin throughout the bone marrow in the treated legs only, suggesting that extracorporeal shock waves induced the bleeding (Fig. 7, $e$ and $f$ ).

At twenty-four hours after extracorporeal shock wave therapy, there were no features of subperiosteal detachment, subperiosteal hemorrhage, or other signs of periosteal damage in the treated tibiae (Fig. 8, $a$ and $b$ ).

\section{Microcrack Analysis}

No differences were found between the treated and untreated tibiae in terms of the microcrack parameters of the trabecular bone samples harvested twenty-four hours after extracorporeal shock wave therapy (Fig. 9). The average crack length was

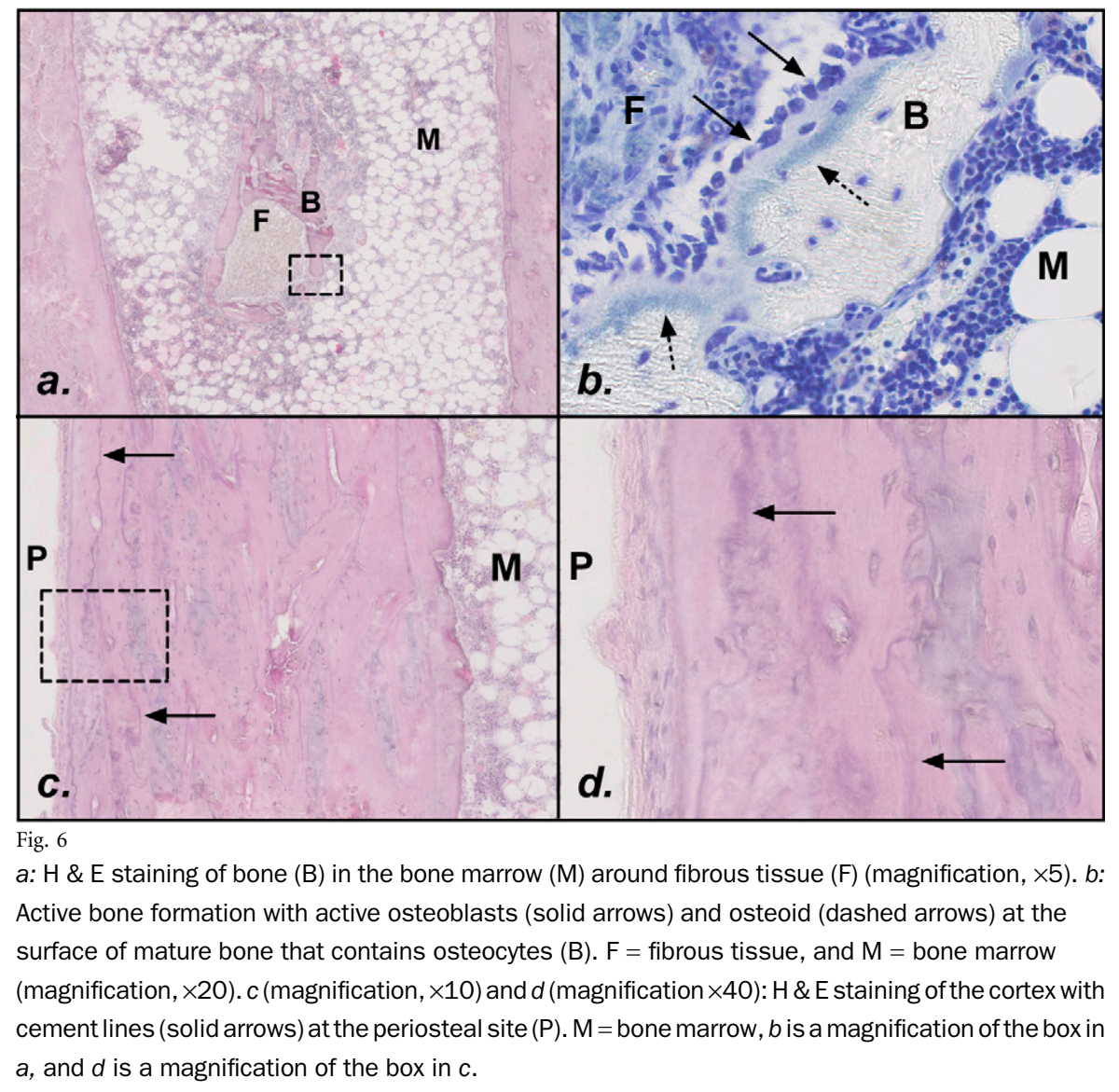


The Journal of Bone \& Joint Surgery $\cdot$ JBJS. Org Volume 93-A • Number $1 \cdot$ January 5, 2011
Unfocused Extracorporeal Shock Waves

Induce Anabolic EfFects in Rat Bone

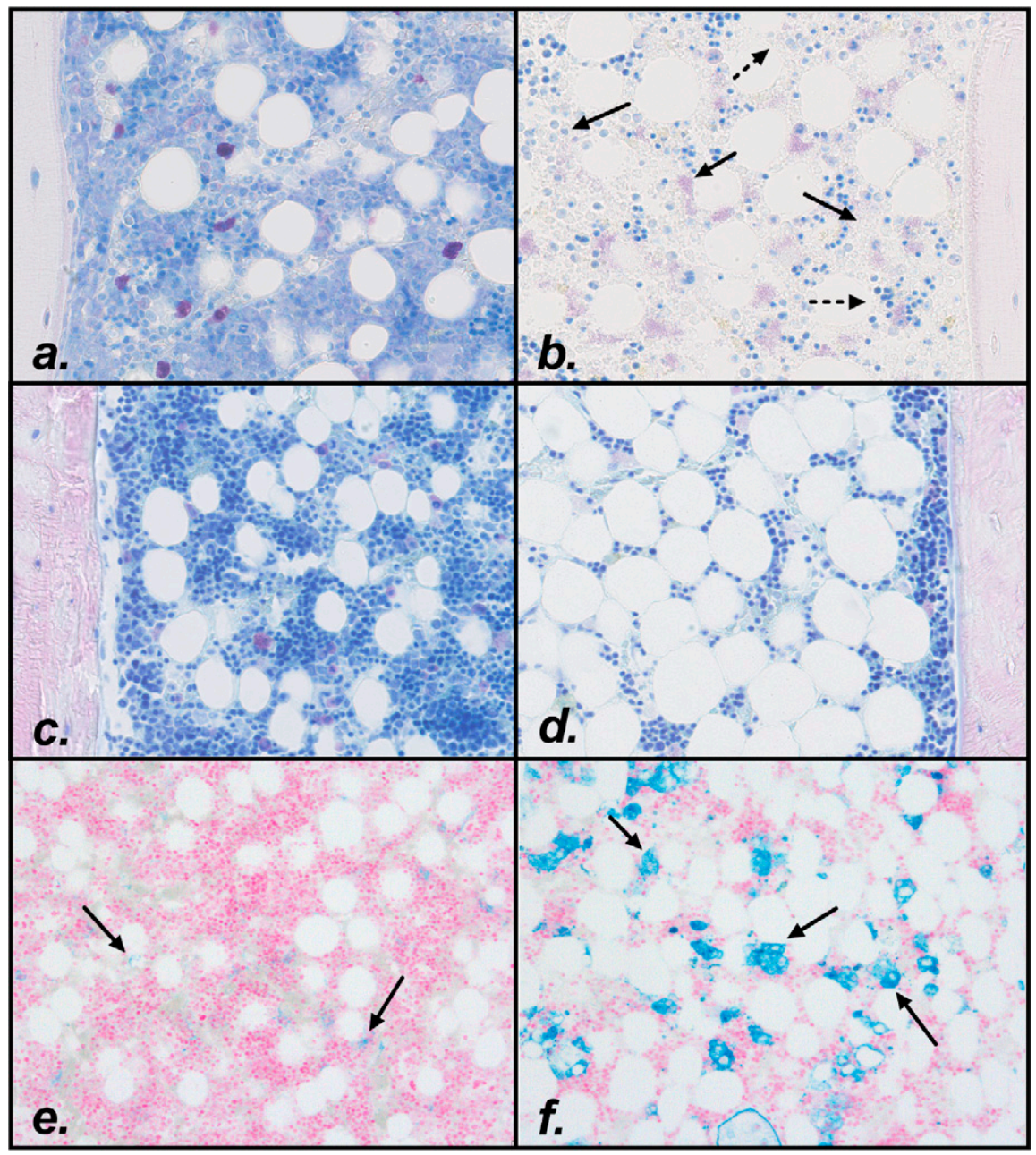

Fig. 7

$a$ and $b$ : May-Grünwald-Giemsa staining of sections of the bone marrow of an untreated control tibia (a) and an extracorporeal shock wave-treated tibia (b) at twenty-four hours after extracorporeal shock wave treatment (magnification, $\times 40$ ). Note the lysis of hematopoietic cells (solid arrows) and the lack of cell membranes around adipocytes (dashed arrows) in the treated leg. $c$ and $d$ : May-Grünwald-Giemsa staining of bone marrow sections of an untreated control tibia (c) and an extracorporeal shock wave-treated tibia (d) at forty-nine days (magnification, $\times 40$ ). Note the hypertrophy of adipocytes. $e$ and $f$ : Perls staining of sections of an untreated control tibia (e) and an extracorporeal shock wave-treated tibia (f) at forty-nine days (magnification, $\times 20$ ). Note the increased presence of hemosiderin (stained blue and indicated by arrows).

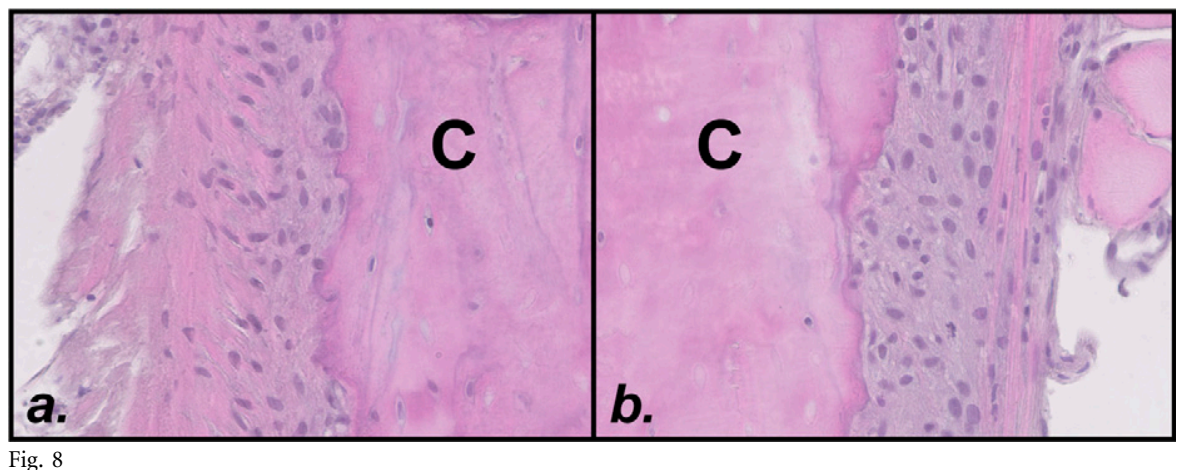

$\mathrm{H} \& \mathrm{E}$ staining of sections of the cortex of a control tibia (a) and an extracorporeal shock wave-treated treated tibia (b) at twenty-four hours after treatment. Subperiosteal bleeding and periosteal detachment were not observed. $\mathrm{C}=$ cortical bone. 

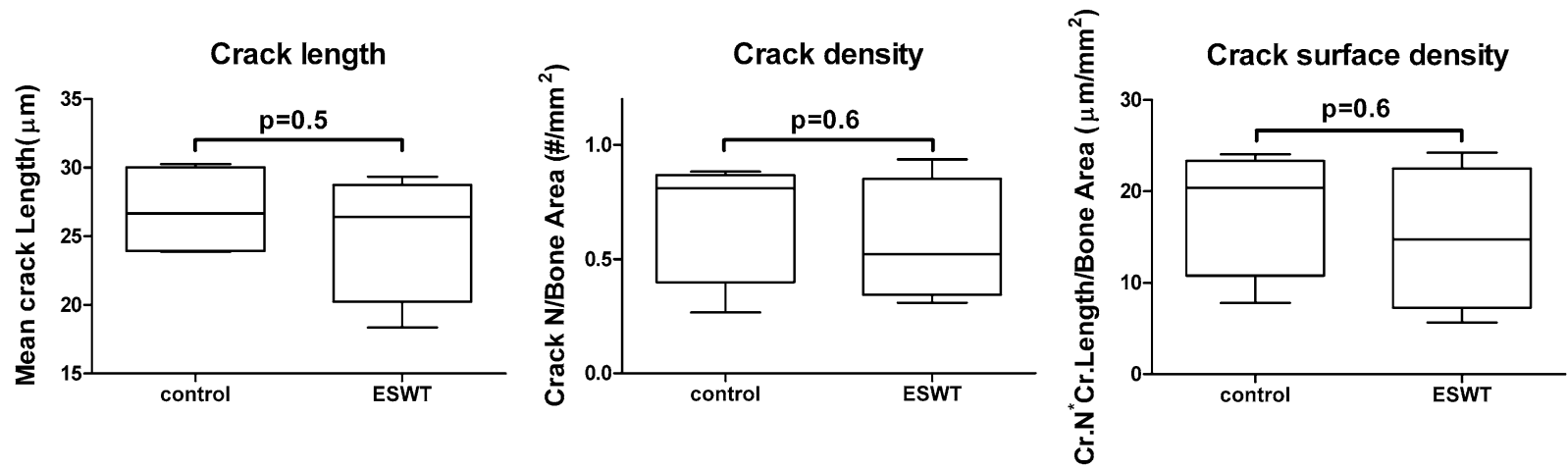

Fig. 9

Results of the microcrack analysis of the control and extracorporeal shock wave-treated (ESWT) tibiae. The mean crack length, crack density, and crack surface density are represented by box-and-whisker plots. The boxes represent the $5 \%$ to $95 \%$ confidence intervals, the horizontal lines within the boxes represent the median, and the I bars represent the minimum and maximum values.

$25.1 \pm 4.7 \mu \mathrm{m}$ in the treated legs and $26.9 \pm 3.4 \mu \mathrm{m}$ in the untreated legs $(\mathrm{p}=0.5)$. The average crack density was $0.57 \pm$ $0.27 \mathrm{crack} / \mathrm{mm}^{2}$ in the treated legs and $0.69 \pm 0.29 \mathrm{crack} / \mathrm{mm}^{2}$ in the untreated legs $(\mathrm{p}=0.6)$. The average crack surface density was $14.8 \pm 7.9 \mu \mathrm{m} / \mathrm{mm}^{2}$ in the treated legs and $18.2 \pm$ $7.1 \mu \mathrm{m} / \mathrm{mm}^{2}$ in the untreated control legs $(\mathrm{p}=0.6)$. No microcracks could be detected in the cortical bone.

\section{Discussion}

$\mathrm{T}$ he present study showed that unfocused extracorporeal shock waves induce anabolic effects in cancellous and cortical rat bone. Use of unfocused extracorporeal shock waves led to bone and bone-marrow damage followed by increased bone formation, increased trabecular bone volume, thicker cortices, formation of de novo trabecular structures, and an increased amount of adipocytes in bone marrow.

Bone formation was followed over time with use of in vivo SPECT scanning of two regions of interest: the metaphysis (consisting mainly of cancellous bone) and the diaphysis (consisting mainly of cortical bone). In both regions of interest, an increased uptake of ${ }^{99 \mathrm{~m}} \mathrm{Tc}-\mathrm{MDP}$ following extracorporeal shock wave therapy was found. The greatest increase of ${ }^{99 \mathrm{~m} T c-M D P}$ uptake at the metaphysis occurred between two and twentyone days after the shock wave therapy, whereas the uptake at the diaphysis was more pronounced at forty-nine days. Other investigators who examined the effects of extracorporeal shock waves on bone turnover reported contradictory findings $^{11,26,27}$; however, because the authors of these latter studies used focused extracorporeal shock waves, different animal models, and conventional bone scintigraphy, it is difficult to make comparisons with the present study.

At forty-nine days after extracorporeal shock wave therapy, our micro-CT analysis showed that extracorporeal shock waves induced new bone formation, resulting in increased trabecular volume fraction, increased connectivity, and more plate-like trabeculae. Furthermore, the trabeculae in the treated tibiae continued to be more distal than those in the control legs, suggesting that extracorporeal shock wave therapy also affected bone remodeling in the distal part of the me- taphysis, where trabeculae are normally resorbed during growth and aging.

CT reconstructions showed ossifications and de novo bone formation in the bone marrow of the diaphysis of all treated legs, whereas no ossification was present in the diaphyseal bone marrow of the untreated, control legs. Because the increased uptake of ${ }^{99 \mathrm{~m}} \mathrm{Tc}-\mathrm{MDP}$ in the diaphysis was mainly at the center, it is likely that the increased uptake reflects these ossifying structures rather than bone formation at the cortices. Histologically, the ossifications were characterized by a mineralized matrix with osteocytes. Along the surface, osteoid with active osteoblasts was found, without cartilaginous tissue, indicating intramembranous bone formation.

Treatment with unfocused extracorporeal shock waves induced damage to the bone marrow, resulting in lysis of hematopoietic cells, destruction of adipocytes, and disruption of microvessels. At forty-nine days, the hematopoietic cells had recovered; however, adipocyte hyperplasia and hypertrophy were still present, probably as a result of the bone-marrow injury. The bone-marrow damage might be related to the finding of fibrous tissue and bone formation. The bone formation always matched with sites of fibrosis. However, from the current findings, it cannot be deduced if (or how) the fibrosis triggers the bone response. We hypothesize that the fibroblasts function as precursor cells and transdifferentiate to osteoblasts, similar to findings reported in distraction osteogenesis ${ }^{28,29}$.

Since microfractures trigger an anabolic response of bone after extracorporeal shock wave therapy, we specifically analyzed the presence of microfractures after the therapy ${ }^{8,10,20}$. However, no differences between the treated and untreated legs were found, making it unlikely that microfractures are responsible for the biological responses.

Periosteal detachment and subperiosteal hemorrhages have been described in studies of focused extracorporeal shock waves $^{10,20,30}$. We noted no evidence of periosteal damage after use of unfocused extracorporeal shock waves. This finding is in line with those of a study of the effect of energy flux densities of focused extracorporeal shock waves on bone ${ }^{20}$. Periosteal detachment was found with use of energy flux densities of 
The Journal of Bone \& Joint Surgery $\cdot$ JbjS. org Volume 93-A • Number $1 \cdot$ January 5, 2011
Unfocused Extracorporeal Shock Waves

Induce Anabolic EfFects in Rat Bone $\geq 0.5 \mathrm{~mJ} / \mathrm{mm}^{2}$, but not with an energy flux density of $0.35 \mathrm{~mJ} / \mathrm{mm}^{2}$. The current study demonstrated that extracorporeal shock wave therapy can induce an anabolic response to the cortical bone without damaging the periosteum. In a previous study, human periosteal cells subjected to low-energy extracorporeal shock waves showed increased proliferation and calcium deposition and a higher viability than cells that received highenergy extracorporeal shock waves ${ }^{31}$.

The present study had several limitations. First, it would have been better if more sequential in vivo micro-CT scans had been made. Future studies should focus on application in an osteoporosis model, evaluate the response of different treatment protocols (different energy flux densities, different numbers of extracorporeal shock waves, different numbers of treatments, etc.), and further elucidate extracorporeal shock wave-induced adipogenesis and other potential side-effects.

Although unfocused extracorporeal shock waves with an energy flux density of $0.1 \mathrm{~mJ} / \mathrm{mm}^{2}$ can be clinically applied without use of analgesics ${ }^{32}$, those with an energy flux density of $0.3 \mathrm{~mJ} / \mathrm{mm}^{2}$ can be painful. Therefore, after we had treated the first two animals, we began giving analgesics prior to extracorporeal shock wave therapy. Intravenous analgesia with nonsteroidal anti-inflammatory drugs prior to extracorporeal shock wave therapy for kidney stones has been reported to provide good suppression of pain ${ }^{33}$. Extracorporeal shock wave therapy itself has an analgesic effect, so the gradual increase of the energy flux density during treatment might also help to reduce pain $^{34,35}$.

In conclusion, a single treatment with unfocused extracorporeal shock waves can increase bone turnover and improve the cancellous and cortical bone architecture as well as the mechanical properties of the treated area. In this experimental setup, extracorporeal shock waves caused damage in the bone marrow, which resulted in hyperplasia and hypertrophy of adipocytes in the bone marrow but did not induce microfractures in the bone or periosteal damage.

Further research is needed to explore potential side effects and the clinical implementation of extracorporeal shock wave therapy for osteoporosis. The anabolic response in both cancellous and cortical bone suggests that unfocused extracorporeal shock waves can potentially be used for local treatment of low bone mass and osteoporosis.

NotE: Tissue Regeneration Technologies provided the shock wave device. The authors thank Prof. J.W. Oosterhuis for his help with the analysis of the histological findings.

O.P. van der Jagt, MD

T.M. Piscaer, MD

N. Kops

H. Jahr, $\mathrm{PhD}$

J.C. van der Linden, $\mathrm{PhD}$

J.H. Waarsing, $\mathrm{PhD}$

J.A.N. Verhaar, MD, PhD

M. de Jong, PhD

$\mathrm{H}$. Weinans, $\mathrm{PhD}$

Departments of Orthopaedics (O.P.v.d.J., T.M.P., N.K., H.J., J.C.v.d.L.,

J.H.W., J.A.N.V., and H.W.) and Nuclear Medicine (M.d.J.), Erasmus MC, University Medical Center Rotterdam, Dr. Molewaterplein 50,

P.O. Box 2040, 3000 CA Rotterdam, The Netherlands.

E-mail address for O.P. van der Jagt: o.vanderjagt@erasmusmc.nl

W. Schaden

AUVA Trauma Center Meidling,

Kundratstrasse 37, 1120 Vienna, Austria

J. $\mathrm{Li}, \mathrm{PhD}$

Department of Anatomy and Cell Biology,

Indiana University School of Medicine,

Indianapolis, IN 46202

\section{References}

1. Warden SJ, Bennell KL, Forwood MR, McMeeken JM, Wark JD. Skeletal effects of low-intensity pulsed ultrasound on the ovariectomized rodent. Ultrasound Med Biol. 2001;27:989-98.

2. Rubin C, Recker R, Cullen D, Ryaby J, McCabe J, McLeod K. Prevention of postmenopausal bone loss by a low-magnitude, high-frequency mechanical stimuli: a clinical trial assessing compliance, efficacy, and safety. J Bone Miner Res. 2004; 19:343-51.

3. Torvinen $\mathrm{S}$, Kannus $\mathrm{P}$, Sievänen $\mathrm{H}$, Järvinen $\mathrm{TA}$, Pasanen $\mathrm{M}$, Kontulainen $\mathrm{S}$, Nenonen A, Järvinen TL, Paakkala T, Järvinen M, Vuori I. Effect of 8-month vertical whole body vibration on bone, muscle performance, and body balance: a randomized controlled study. J Bone Miner Res. 2003;18:876-84.

4. Tabrah F, Hoffmeier M, Gilbert F Jr, Batkin S, Bassett CA. Bone density changes in osteoporosis-prone women exposed to pulsed electromagnetic fields (PEMFs). J Bone Miner Res. 1990;5:437-42.

5. Wang $\mathrm{CJ}$, Huang $\mathrm{HY}$, Chen $\mathrm{HH}$, Pai $\mathrm{CH}$, Yang KD. Effect of shock wave therapy on acute fractures of the tibia: a study in a dog model. Clin Orthop Relat Res. 2001;387:112-8.

6. Rompe JD, Rosendahl T, Schöllner C, Theis C. High-energy extracorporeal shock wave treatment of nonunions. Clin Orthop Relat Res. 2001;387:102-11.

7. Schaden W, Fischer A, Sailler A. Extracorporeal shock wave therapy of nonunion or delayed osseous union. Clin Orthop Relat Res. 2001;387:90-4.

8. Valchanou VD, Michailov P. High energy shock waves in the treatment of delayed and nonunion of fractures. Int Orthop. 1991;15:181-4.
9. Ogden JA, Tóth-Kischkat A, Schultheiss R. Principles of shock wave therapy. Clin Orthop Relat Res. 2001;387:8-17.

10. Delius M, Draenert K, Al Diek Y, Draenert Y. Biological effects of shock waves: in vivo effect of high energy pulses on rabbit bone. Ultrasound Med Biol. 1995;21: 1219-25.

11. Maier M, Milz S, Tischer T, Münzing W, Manthey N, Stäbler A, Holzknecht N, Weiler C, Nerlich A, Refior HJ, Schmitz C. Influence of extracorporeal shock-wave application on normal bone in an animal model in vivo. Scintigraphy, MRI and histopathology. J Bone Joint Surg Br. 2002;84:592-9.

12. Wang $\mathrm{CJ}$, Yang KD, Wang FS, Hsu CC, Chen $\mathrm{HH}$. Shock wave treatment shows dose-dependent enhancement of bone mass and bone strength after fracture of the femur. Bone. 2004;34:225-30.

13. Chen YJ, Wurtz T, Wang CJ, Kuo YR, Yang KD, Huang HC, Wang FS. Recruitment of mesenchymal stem cells and expression of TGF-beta 1 and VEGF in the early stage of shock wave-promoted bone regeneration of segmental defect in rats. J Orthop Res. 2004;22:526-34.

14. Wang FS, Yang KD, Chen RF, Wang CJ, Sheen-Chen SM. Extracorporeal shock wave promotes growth and differentiation of bone-marrow stromal cells towards osteoprogenitors associated with induction of TGF-beta1. J Bone Joint Surg Br. 2002;84:457-61.

15. Wang FS, Yang KD, Kuo YR, Wang CJ, Sheen-Chen SM, Huang HC, Chen YJ. Temporal and spatial expression of bone morphogenetic proteins in extracorpo- 
The Journal of Bone \& Joint Surgery $\cdot$ JBJS. Org Volume 93-A • Number $1 \cdot$ January 5, 2011
Unfocused Extracorporeal Shock Waves

Induce ANABolic EFFects in Rat Bone real shock wave-promoted healing of segmental defect. Bone. 2003;32: 387-96.

16. Wang CJ, Wang FS, Ko JY, Huang HY, Chen CJ, Sun YC, Yang YJ. Extracorporeal shockwave therapy shows regeneration in hip necrosis. Rheumatology (Oxford). 2008;47:542-6.

17. Wang FS, Wang CJ, Sheen-Chen SM, Kuo YR, Chen RF, Yang KD. Superoxide mediates shock wave induction of ERK-dependent osteogenic transcription factor (CBFA1) and mesenchymal cell differentiation toward osteoprogenitors. J Biol Chem. 2002;277:10931-7.

18. Wang FS, Yang KD, Wang CJ, Huang HC, Chio CC, Hsu TY, Ou CY. Shockwave stimulates oxygen radical-mediated osteogenesis of the mesenchymal cells from human umbilical cord blood. J Bone Miner Res. 2004;19:973-82

19. Kaulesar Sukul DM, Johannes EJ, Pierik EG, van Eijck GJ, Kristelijn MJ. The effect of high energy shock waves focused on cortical bone: an in vitro study. J Surg Res. 1993;54:46-51.

20. Tischer T, Milz S, Weiler C, Pautke C, Hausdorf J, Schmitz C, Maier M. Dosedependent new bone formation by extracorporeal shock wave application on the intact femur of rabbits. Eur Surg Res. 2008;41:44-53.

21. Beekman $F$, van der Have $F$. The pinhole: gateway to ultra-high-resolution threedimensional radionuclide imaging. Eur J Nucl Med Mol Imaging. 2007;34:151-61.

22. Erasmus MC. The Erasmus Orthopaedic Research Laboratory. www. erasmusmc.nl/orthopaedie/research/labor/downloads/?lang=nl. Accessed 2009 Mar 11.

23. Waarsing JH, Day JS, Weinans $\mathrm{H}$. An improved segmentation method for in vivo microCT imaging. J Bone Miner Res. 2004;19:1640-50.

24. Westbroek I, Waarsing JH, van Leeuwen JP, Waldum $\mathrm{H}$, Reseland JE, Weinans $\mathrm{H}$, Syversen U, Gustafsson BI. Long-term fluoxetine administration does not result in major changes in bone architecture and strength in growing rats. J Cell Biochem. 2007:101:360-8.

25. Burr DB, Hooser M. Alterations to the en bloc basic fuchsin staining protocol for the demonstration of microdamage produced in vivo. Bone. 1995;17:431-3.

26. Verna M, Turner TA, Anderson KL. Scintigraphic, radiographic, and thermographic appearance of the metacarpal and metatarsal regions of adult healthy horses treated with nonfocused extracorporeal shock wave therapy-a pilot study. Vet Ther. 2005;6:268-76.

27. Bischofberger AS, Ringer SK, Geyer H, Imboden I, Ueltschi G, Lischer CJ. Histomorphologic evaluation of extracorporeal shock wave therapy of the fourth metatarsal bone and the origin of the suspensory ligament in horses without lameness. Am J Vet Res. 2006;67:577-82.

28. Perrien DS, Brown EC, Aronson J, Skinner RA, Montague DC, Badger TM, Lumpkin CK Jr. Immunohistochemical study of osteopontin expression during distraction osteogenesis in the rat. J Histochem Cytochem. 2002;50:567-74.

29. Yasui N, Sato M, Ochi T, Kimura T, Kawahata H, Kitamura Y, Nomura S. Three modes of ossification during distraction osteogenesis in the rat. J Bone Joint Surg Br. 1997;79:824-30

30. Takahashi K, Yamazaki M, Saisu T, Nakajima A, Shimizu S, Mitsuhashi S, Moriya H. Gene expression for extracellular matrix proteins in shockwave-induced osteogenesis in rats. Calcif Tissue Int. 2004;74:187-93.

31. Tam KF, Cheung WH, Lee KM, Qin L, Leung KS. Delayed stimulatory effect of low-intensity shockwaves on human periosteal cells. Clin Orthop Relat Res. 2005;438:260-5

32. Schaden W, Thiele R, Kölpl C, Pusch M, Nissan A, Attinger CE, ManiscalcoTheberge ME, Peoples GE, Elster EA, Stojadinovic A. Shock wave therapy for acute and chronic soft tissue wounds: a feasibility study. J Surg Res. 2007;143:1-12.

33. Takmaz SA, Inan N, Goktug A, Erdogan I, Sunay M, Ceyhan A. The analgesic effect of 8 and $16 \mathrm{mg}$ lornoxicam administered before shock wave lithotripsy: a randomized, double-blind, controlled study. Urology. 2008;72:282-5.

34. Ochiai N, Ohtori S, Sasho T, Nakagawa K, Takahashi K, Takahashi N, Murata R Takahashi K, Moriya H, Wada Y, Saisu T. Extracorporeal shock wave therapy improves motor dysfunction and pain originating from knee osteoarthritis in rats. Osteoarthritis Cartilage. 2007;15:1093-6.

35. Takahashi N, Wada Y, Ohtori S, Saisu T, Moriya H. Application of shock waves to rat skin decreases calcitonin gene-related peptide immunoreactivity in dorsal root ganglion neurons. Auton Neurosci. 2003;107:81-4. 